\title{
Asymptotic Critical Transmission Radius and Critical Neighbor Number for $k$-Connectivity in Wireless Ad Hoc Networks
}

\author{
Peng-Jun Wan Chih-Wei Yi \\ Department of Computer Science, Illinois Institute of Technology \\ 10 West 31st Street \\ Chicago, IL 60616, USA. \\ Emails: wan@cs.iit.edu, yichihw@iit.edu
}

\begin{abstract}
A range assignment to the nodes in a wireless ad hoc network induces a topology in which there is an edge between two nodes if and only if both of them are within each other's transmission range. The critical transmission radius for $k$ connectivity is the smallest $r$ such that if all nodes have the transmission radius $r$, the induced topology is $k$-connected. The critical neighbor number for $k$-connectivity is the smallest integer $l$ such that if every node sets its transmission radius equal to the distance between itself and its $l$-th nearest neighbor, the induced topology is $k$-connected. In this paper, we study the asymptotic critical transmission radius for $k$-connectivity and asymptotic critical neighbor number for $k$-connectivity in a wireless ad hoc network whose nodes are uniformly and independently distributed in a unit-area square or disk. We provide a precise asymptotic distribution of the critical transmission radius for $k$-connectivity and an improved asymptotic almost sure upper bound on the critical neighbor number for $k$-connectivity.
\end{abstract}

\section{Categories and Subject Descriptors}

C.2.1 [Network Architecture and Design]: Network topology; Wireless communication; C.4 [Performance of Systems]: Fault tolerance; Reliability, availability, and serviceability; G.2.2 [Graph Theory]: Network problems

\section{General Terms}

Performance, Reliability, Theory

\section{Keywords}

Random geometric graph, critical transmission radius, critical neighbor number, asymptotic distribution.

Permission to make digital or hard copies of all or part of this work for personal or classroom use is granted without fee provided that copies are not made or distributed for profit or commercial advantage and that copies bear this notice and the full citation on the first page. To copy otherwise, to republish, to post on servers or to redistribute to lists, requires prior specific permission and/or a fee.

MobiHoc'04, May 24-26, 2004, Roppongi, Japan.

Copyright 2004 ACM 1-58113-849-0/04/0005 ...\$5.00.

\section{INTRODUCTION}

Let $V$ be the set of radio nodes in a wireless ad hoc network. A range assignment to $V$ specifies a transmission radius to each node $v$ in $V$. The network topology induced by a range assignment is a graph on $V$ with an edge connecting each pair of nodes whose distance is no more than either of their transmission radii. There are two simple range assignment schemes which are both completely determined by a single parameter. In a uniform range assignment with a parameter $r>0$, every node has the same transmission radius of $r$. The network topology induced by this range assignment, denoted by $G_{r}(V)$, is the $r$-graph on $V$ in which each pair of nodes separated by a distance of at most $r$ is connected by an edge. In a $l$-nearest-neighbor range assignment with an integer parameter $l>0$, every node sets its transmission radius equal to the distance between itself and its $l$-th nearest neighbor. The network topology induced by this range assignment, denoted by $H_{l}(V)$, is the symmetric $l$-nearest-neighbor graph on $V$ in which there is an edge between each pair of nodes which are both one of each other's $l$ nearest neighbors.

In general, a range assignment has to ensure that certain topological properties are met by the induced network topology. Two topological properties of interest are $k$-connectivity and vertex degree at least $k$. Let $\kappa$ and $\delta$ denote the connectivity and the smallest vertex degree respectively of a graph. Then these two properties can be simply represented by $\kappa \geq k$ and $\delta \geq k$ respectively. Both properties are monotone-increasing, which means that all supergraphs of a graph with these properties also have these properties as well. For a monotone-increasing topological property $Q$, the critical (or hitting) transmission radius, denoted by $\rho(V ; Q)$, is the smallest $r$ at which $G_{r}(V)$ has property $Q$, and the critical (or hitting) neighbor number, denoted by $\ell(V ; Q)$, is the smallest $l$ at which $H_{l}(V)$ has property $Q$. Note that $\rho(V ; Q)$ is always the distance between some pair of nodes, and $\ell(V ; Q)$ is always an integer no more than the size of $V$. Thus, for those $Q$ which can be tested in polynomial time (such as $\kappa \geq k$ and $\delta \geq k$ ), both $\rho(V ; Q)$ and $\ell(V ; Q)$ can be obtained in polynomial time as well.

This paper is concerned with the asymptotic critical transmission radius and critical neighbor number for a desired 
connectivity in a random wireless ad hoc network. Specifically, the radio devices are represented by a uniform $n$-point process $\mathcal{X}_{n}$ over a unit-area region $\Omega$, i.e., a set of $n$ independent points each of which is uniformly distributed over $\Omega$. Then both $G_{r}\left(\mathcal{X}_{n}\right)$ and $H_{l}\left(\mathcal{X}_{n}\right)$ are random graphs, and both $\rho\left(\mathcal{X}_{n} ; \kappa \geq k\right)$ and $\ell\left(\mathcal{X}_{n} ; \kappa \geq k\right)$ are random variables. In this paper, the region $\Omega$ is assumed to be either a disk or a square. For such $\Omega$, we provide a precise asymptotic distribution of $\rho\left(\mathcal{X}_{n} ; \kappa \geq k\right)$ and an asymptotic almost sure upper bound on $\ell\left(\mathcal{X}_{n} ; \kappa \geq k\right)$ when $n$ goes to infinity. The latter upper bound improves the previous known bound.

In what follows, $\|x\|$ is the Euclidean norm of a point $x \in \mathbb{R}^{2}$, and $|A|$ is shorthand for 2-dimensional Lebesgue measure (or area) of a measurable set $A \subset \mathbb{R}^{2}$. All integrals considered will be Lebesgue integrals. The topological boundary of a set $A \subset \mathbb{R}^{2}$ is denoted by $\partial A$. The disk of radius $r$ centered at $x$ is denoted by $D(x, r)$. An event is said to be asymptotic almost sure (abbreviated by a.a.s.) if it occurs with a probability converges to one as $n \rightarrow \infty$. The symbols $O, o, \sim$ always refer to the limit $n \rightarrow \infty$. To avoid trivialities, we tacitly assume $n$ to be sufficiently large if necessary. For simplicity of notation, the dependence of sets and random variables on $n$ will be frequently suppressed.

The remaining of this paper is organized as follows. In Section 2, we briefly describe related works. In Section 3, we give the precise asymptotic distribution of $\rho\left(\mathcal{X}_{n} ; \kappa \geq k\right)$. In Section 4, we present an improved asymptotic almost sure upper bound on $\ell\left(\mathcal{X}_{n} ; \kappa \geq k\right)$. Finally, we conclude this paper in Section 5.

\section{RELATED WORKS}

Since $\kappa \geq k$ implies that $\delta \geq k, \rho\left(\mathcal{X}_{n} ; \kappa \geq k\right)$ is always at least $\rho\left(\mathcal{X}_{n} ; \delta \geq k\right)$. A fascinating result proved by Penrose [13] [14] states that they are equal a.a.s.. This means when $n$ is big enough, then with high probability, if one starts with isolated points and adds edges connecting the points of $\mathcal{X}_{n}$ in order of increasing length, then the resulting graph becomes $k$-connected as soon as the last vertex of degree $k-1$ vanishes. Thus, $\rho\left(\mathcal{X}_{n} ; \kappa \geq k\right)$ and $\rho\left(\mathcal{X}_{n} ; \delta \geq k\right)$ have the same asymptotic distribution. Although Penrose [13] [14] considered only $\mathcal{X}_{n}$ over a unit-area square, the same result can be extended to $\mathcal{X}_{n}$ over a unit-area disk as well with proper modification.

For $k=1$ and $\mathcal{X}_{n}$ over a unit-area square, the precise asymptotic distribution of $\rho\left(\mathcal{X}_{n} ; \delta \geq 1\right)$ has been derived by Dette and Henze [3] much earlier: for any constant $c$,

$$
\operatorname{Pr}\left\{\rho\left(\mathcal{X}_{n} ; \delta \geq 1\right) \leq \sqrt{\frac{\log n+c}{\pi n}}\right\} \sim \exp \left(-e^{-c}\right) .
$$

The same asymptotic distribution also holds for $\mathcal{X}_{n}$ over a unit-area disk. For $k>1$, Penrose [14] presented the following limiting property of $\rho\left(\mathcal{X}_{n} ; \delta \geq k\right)$ for $\Omega$ being a unit-area square, which also holds for $\bar{\Omega}$ being a unit-area disk.
Theorem 1. [14] Let $k>0$ and $\lambda \in \mathbb{R}$. Then for any sequence $\left(r_{n}\right)_{n \geq 1}$ satisfying

$$
\frac{n}{k !} \int_{\Omega}\left(n\left|D\left(x, r_{n}\right) \cap \Omega\right|\right)^{k} e^{-n\left|D\left(x, r_{n}\right) \cap \Omega\right|} d x \sim \lambda,
$$

the probabilities of the two events $\rho\left(\mathcal{X}_{n} ; \delta \geq k+1\right) \leq r_{n}$ and $\rho\left(\mathcal{X}_{n} ; \kappa \geq k+1\right) \leq r_{n}$ both converge to $e^{-\lambda}$ as $n \rightarrow \infty$.

A better understanding of Theorem 1 necessitates a brief explanation of the Poissonization technique used by Penrose [14] for the proof. Let $\mathcal{P}_{n}$ denote a homogeneous Poisson process of intensity $n$ on $\Omega$. Recall that $\mathcal{P}_{n}$ is characterized by the following property: if $A_{1}, A_{2}, \cdots, A_{m}$ are arbitrarily disjoint regions of $\Omega$, then the numbers of points in $\mathcal{P}_{n}$ on $A_{1}, A_{2}, \cdots, A_{m}$ are mutually independent Poisson random variables with intensity $n\left|A_{1}\right|, n\left|A_{2}\right|, \cdots, n\left|A_{m}\right|$, respectively. The relevance of $\mathcal{P}_{n}$ to $\mathcal{X}_{n}$ is that given that there are exactly $k$ points of $\mathcal{P}_{n}$ in a region $A \Omega$, these $k$ points are independently and uniformly distributed in $A$. Thus, $\mathcal{X}_{n}$ can be well approximated by $\mathcal{P}_{n}$. Due to the extreme independence property, $\mathcal{P}_{n}$ is much more convenient to be dealt with. Penrose [14] thus first proved a Poissonized version of Theorem 1 in which $\mathcal{P}_{n}$ is replaced by $\mathcal{X}_{n}$, and then de-Poissonize this Poissonized version to complete the proof of Theorem 1 . The value

$$
\frac{n}{k !} \int_{\Omega}\left(n\left|D\left(x, r_{n}\right) \cap \Omega\right|\right)^{k} e^{-n\left|D\left(x, r_{n}\right) \cap \Omega\right|} d x
$$

in Theorem 1 is exactly the expected number of points of $\mathcal{P}_{n}$ with degree $k$ in $G_{r_{n}}\left(\mathcal{P}_{n}\right)$. The value $\lambda$ is thus the limit of the expected number of points of $\mathcal{P}_{n}$ with degree $k$ in $G_{r_{n}}\left(\mathcal{P}_{n}\right)$.

However, Penrose [14] didn't provide the explicit form of $r_{n}$, while stating that $r_{n}$ is not so easy to find because of the dominance of complicated boundary effect. To explain the boundary effect, we define the $r$-neighborhood of a point $x$ as $D(x, r) \cap \Omega$. The area of such $r$-neighborhood of a point in $\mathcal{X}_{n}$ determines the distribution of the number of neighbors in $G_{r}\left(\mathcal{X}_{n}\right)$. The larger this area, the higher the expected number of neighbors. As a node close to the boundary of $\Omega$ has small $r$-neighborhood, intuitively a node around the boundary have smaller vertex degree. On the other hand, the probability for a node to be around the boundary is small when the node density is large. The overall effect produced by the boundary nodes is thus complicated and even peculiar [4]. In this paper, we will present a partition of $\Omega$ to address the boundary effect, based on which we obtain the explicit form of $r_{n}$.

A tight analytical result on asymptotic critical transmission radius for connectivity can be found in [6], and other earlier simulation studies and/or loose analytical results on asymptotic critical transmission radius for connectivity can be found in [1] [2] [5] [10] [12] [15] [16] [19].

The problem of how many neighbors is desirable for various purposes in a wireless ad hoc network whose nodes are specified by a planar Poisson point process has been studied since the 1970s. For the purpose of maximizing the one-hop progress of a packet in the desired direction under the slotted ALOHA protocol, Kleinrock and Silvester [9] proposed 
that if all nodes have the same transmission power then six was the "magic number", i.e., on average every node should connect itself to its six nearest neighbors. Later, the magic number was revised to eight by Takagi and Kleinrock [18]. The same paper [18] also considered other transmission protocols, which resulted in some other magic numbers five and seven. Hou and $\mathrm{Li}[8]$ considered the situation when each node is allowed to adjust its transmission range individually, and obtained the magic numbers six and eight. For the purpose of maximizing the transmission efficiency defined as the ratio between the expected progress and the area covered by the transmission, Hajek [7] suggested that each node should adjust its power to cover about three nearest neighbors on average. Mathar and Mattfeldt [11] analyzed the wireless network generated by a Poisson point process on a line, and also obtained some magic numbers.

However, none of the analyses in [7] [8] [9] [11] [18] took connectivity into consideration. Based on simulations, $\mathrm{Ni}$ and Chandler [12] suggested that six to eight nearest neighbors can make a small size network connected with high probability. But it turns out that as the number of nodes in the network increases, the network becomes disconnected with probability one whether one connects to six or eight nearest neighbors. In fact, Xue and Kumar [20] recently proved that even if each node connects to $0.074 \log n$ nearest neighbors the probability that the network is disconnected converges to one as the numbers of nodes in the network increases. On the other hand, if each node connects to greater than $5.1774 \log n$ nearest neighbors, then the network is asymptotically connected. Using the dePoissonization technique, Xue and Kumar [20] proved that $0.074 \log n$ and $5.1774 \log n$ are a.a.s. lower bound and upper bound respectively on $\ell\left(\mathcal{X}_{n} ; \kappa \geq 1\right)$. In this paper, we prove that for any $k \geq 1$ and any real number $\alpha>1$, $\alpha e \log n$ is an upper bound on $\ell\left(\mathcal{X}_{n} ; \kappa \geq k\right)$, where $e \approx 2.718$ is the natural base.

\section{CRITICAL TRANSMISSION RADIUS FOR $K$-CONNECTIVITY}

The main results of this section are the following two theorems.

TheOREM 2. Assume that $\Omega$ is the unit-area square. Let

$$
r_{n}=\sqrt{\frac{\log n+(2 k-1) \log \log n+\xi}{\pi n}}
$$

where

$$
\xi= \begin{cases}-2 \log \left(\sqrt{e^{-c}+\frac{\pi}{4}}-\frac{\sqrt{\pi}}{2}\right) & \text { if } k=1, \\ 2 \log \frac{\sqrt{\pi}}{2^{k-1} k !}+2 c & \text { if } k>1 .\end{cases}
$$

Then the probabilities of the two events $\rho\left(\mathcal{X}_{n} ; \delta \geq k+1\right) \leq$ $r_{n}$ and $\rho\left(\mathcal{X}_{n} ; \kappa \geq k+1\right) \leq r_{n}$ both converge to $\exp \left(-e^{-\bar{c}}\right)$ as $n \rightarrow \infty$.
Theorem 3. Assume that $\Omega$ is the unit-area disk. Let

$$
r_{n}=\sqrt{\frac{\log n+(2 k-1) \log \log n+\xi}{\pi n}},
$$

where

$$
\xi= \begin{cases}-2 \log \left(\sqrt{e^{-c}+\frac{\pi^{2}}{16}}-\frac{\pi}{4}\right) & \text { if } k=1 ; \\ 2 \log \frac{\pi}{2^{k} k !}+2 c & \text { if } k>1 .\end{cases}
$$

Then the probabilities of the two events $\rho\left(\mathcal{X}_{n} ; \delta \geq k+1\right) \leq$ $r_{n}$ and $\rho\left(\mathcal{X}_{n} ; \kappa \geq k+1\right) \leq r_{n}$ both converge to $\exp \left(-e^{-\bar{c}}\right)$ as $n \rightarrow \infty$.

Throughout of this section, we use $r_{n}$ to denote the value given either in Theorem 2 or in Theorem 3 depending on whether $\Omega$ is a square or a disk. For any $t \in\left[0, r_{n}\right]$, let $a_{n}(t)$ denote the area of the region

$$
\left\{x=\left(x_{1}, x_{2}\right): x_{1}^{2}+x_{2}^{2} \leq r_{n}^{2}, x_{1} \leq t\right\}
$$

(see Figure 1). It is easy to see that $a_{n}^{\prime}(t)$ equals to length of the boundary chord, i.e., $2 \sqrt{r_{n}^{2}-t^{2}}$. For simplicity, we will omit all subscript $n$.

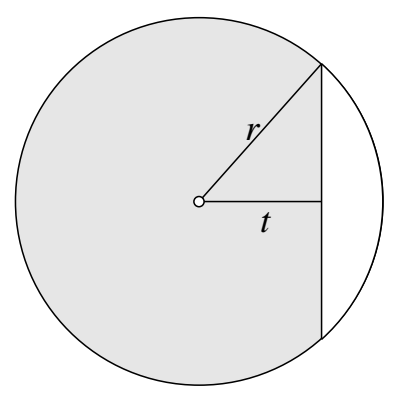

Figure 1: The area of the shaded region is $a_{n}(t)$.

We first present the following technical lemma.

LEMMA 4. $n \int_{0}^{\frac{r}{2}} \frac{(n a(t))^{k} e^{-n a(t)}}{k !} d t \sim \frac{\sqrt{\pi}}{2^{k+1} k !} e^{-\frac{\xi}{2}}$.

PROOF. It is straightforward to verify that

$$
\frac{1}{r}\left(n \pi r^{2}\right)^{k} e^{-\frac{n \pi r^{2}}{2}} \sim \sqrt{\pi} e^{-\frac{\xi}{2}} .
$$

Let $f(t)=n a(t)$. Using integration by parts on the integral yields

$$
\begin{aligned}
& n \int_{0}^{\frac{r}{2}} \frac{(f(t))^{k} e^{-f(t)}}{k !} d t \\
& =n \int_{0}^{\frac{r}{2}} \frac{(f(t))^{k} e^{-f(t)}}{k !} \frac{1}{n a^{\prime}(t)} d f(t) \\
& =\int_{0}^{\frac{r}{2}} \frac{1}{a^{\prime}(t)} d\left(-e^{-f(t)} \sum_{i=0}^{k} \frac{(f(t))^{i}}{i !}\right) \\
& =-\left.\frac{1}{a^{\prime}(t)} e^{-f(t)} \sum_{i=0}^{k} \frac{(f(t))^{i}}{i !}\right|_{0} ^{\frac{r}{2}} \\
& \quad-\int_{0}^{\frac{r}{2}} \frac{a^{\prime \prime}(t)}{\left(a^{\prime}(t)\right)^{2}} e^{-f(t)} \sum_{i=0}^{k} \frac{(f(t))^{i}}{i !} d t
\end{aligned}
$$


The first term is asymptotically equal to $\frac{\sqrt{\pi}}{2^{k+1} k !} e^{-\frac{\xi}{2}}$ because

$$
\begin{aligned}
& \frac{1}{a^{\prime}\left(\frac{r}{2}\right)} e^{-f\left(\frac{r}{2}\right)} \sum_{i=0}^{k} \frac{\left(f\left(\frac{r}{2}\right)\right)^{i}}{i !} \\
& =\frac{1}{\sqrt{3} r} e^{-\frac{5 n \pi r^{2}}{6}} \sum_{i=0}^{k} \frac{\left(\frac{5 n \pi r^{2}}{6}\right)^{i}}{i !}=o(1),
\end{aligned}
$$

and

$$
\begin{aligned}
& \frac{1}{a^{\prime}(0)} e^{-f(0)} \sum_{i=0}^{k} \frac{(f(0))^{i}}{i !} \\
& =\frac{1}{2 r} e^{-\frac{n \pi r^{2}}{2}} \sum_{i=0}^{k} \frac{\left(\frac{n \pi r^{2}}{2}\right)^{i}}{i !} \\
& \sim \frac{1}{2^{k+1} k !} \frac{1}{r} e^{-\frac{n \pi r^{2}}{2}}\left(n \pi r^{2}\right)^{k}+o(1) \\
& \sim \frac{\sqrt{\pi}}{2^{k+1} k !} e^{-\frac{\xi}{2}} .
\end{aligned}
$$

The second term is asymptotically negligible because

$$
\begin{aligned}
& \left|\int_{0}^{\frac{r}{2}} \frac{a^{\prime \prime}(t)}{\left(a^{\prime}(t)\right)^{2}} e^{-f(t)} \sum_{i=0}^{k} \frac{(f(t))^{i}}{i !} d t\right| \\
& =\left|\frac{1}{n} \int_{0}^{\frac{r}{2}} \frac{a^{\prime \prime}(t)}{\left(a^{\prime}(t)\right)^{3}} e^{-f(t)} \sum_{i=0}^{k} \frac{(f(t))^{i}}{i !} d f(t)\right| \\
& =\frac{1}{4 n} \int_{0}^{\frac{r}{2}} \frac{t}{\left(r^{2}-t^{2}\right)^{2}} e^{-f(t)} \sum_{i=0}^{k} \frac{(f(t))^{i}}{i !} d f(t) \\
& \leq \frac{1}{8 n r^{3}} \int_{0}^{\frac{r}{2}} e^{-f(t)} \sum_{i=0}^{k} \frac{(f(t))^{i}}{i !} d f(t) \\
& \leq O(1) \frac{1}{n r^{3}} \int_{0}^{\frac{r}{2}} e^{-f(t)} \frac{(f(t))^{k}}{k !} d f(t) \\
& =O(1) \frac{1}{n r^{3}} \int_{0}^{\frac{r}{2}} d\left(-e^{-f(t)} \sum_{i=0}^{k} \frac{(f(t))^{i}}{i !}\right) \\
& \leq O(1) \frac{1}{n r^{3}} e^{-f(0)} \sum_{i=0}^{k} \frac{(f(0))^{i}}{i !} \\
& =O(1) \frac{1}{n r^{2}}\left(\frac{1}{r} e^{-f(0)} \sum_{i=0}^{k} \frac{(f(0))^{i}}{i !}\right) \\
& =O(1) \frac{1}{n r^{2}}=o(1) .
\end{aligned}
$$

Thus, the lemma follows.

In the next two subsections, we give the proofs for Theorem 2 and Theorem 3 respectively.

\subsection{Proof for Theorem 2}

By Theorem 1, we only need to show that

$$
\frac{n}{k !} \int_{\Omega}(n|D(x, r) \cap \Omega|)^{k} e^{-n|D(x, r) \cap \Omega|} d x \sim e^{-c} .
$$

To address the boundary effect of the square region $\Omega$, we partition $\Omega$ into three subregions $\Omega(0), \Omega(1)$ and $\Omega(2)$ as illustrated in Figure 2. For any $0 \leq i \leq 2$, let $\Omega(i)$ denote the set of $x \in \Omega$ satisfying that $D(x, r)$ intersects exactly $i$ sides of $\Omega$. The areas of these three regions are

$$
\begin{aligned}
& |\Omega(0)|=(1-2 r)^{2}, \\
& |\Omega(1)|=4 r(1-2 r), \\
& |\Omega(2)|=4 r^{2} .
\end{aligned}
$$

For any $x \in \Omega(i)$,

$$
|D(x, r) \cap \Omega| \geq 2^{-i} \pi r^{2} .
$$

When $x \in \Omega(1),|D(x, r) \cap \Omega|$ is exactly $a(t)$, where $t$ is the distance between $x$ and the boundary of $\Omega$.

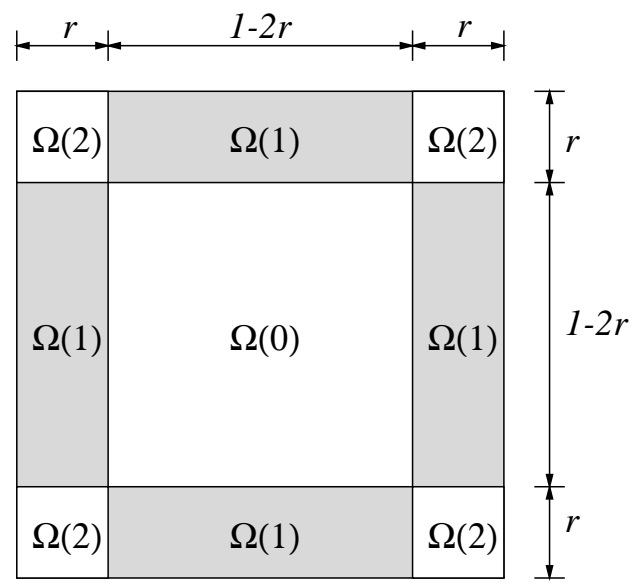

Figure 2: Parition of the square $\Omega$.

First, we calculate the integration over $\Omega(0)$. If $x \in \Omega(0)$, $|D(x, r) \cap \Omega|=\pi r^{2}$. Thus,

$$
\begin{aligned}
& \frac{n}{k !} \int_{\Omega(0)}(n|D(x, r) \cap \Omega|)^{k} e^{-n|D(x, r) \cap \Omega|} d x \\
& =\frac{n}{k !}\left(n \pi r^{2}\right)^{k} e^{-n \pi r^{2}}|\Omega(0)| \\
& \sim \frac{n}{k !}\left(n \pi r^{2}\right)^{k} e^{-n \pi r^{2}} \\
& \sim \begin{cases}e^{-\xi} & \text { if } k=1, \\
o(1) & \text { if } k>1 .\end{cases}
\end{aligned}
$$

Now, we calculate the integration over $\Omega(2)$. If $x \in \Omega(2)$,

$$
|D(x, r) \cap \Omega| \geq \frac{1}{4} \pi r^{2} .
$$

Thus,

$$
\begin{aligned}
& \frac{n}{k !} \int_{\Omega(2)}(n|D(x, r) \cap \Omega|)^{k} e^{-n|D(x, r) \cap \Omega|} d x \\
& \leq \frac{n}{k !}\left(n \pi r^{2}\right)^{k} e^{-\frac{n \pi r^{2}}{4}}|\Omega(2)| \\
& =O(1)\left(n \pi r^{2}\right)^{k+1} e^{-\frac{n \pi r^{2}}{4}}=o(1) .
\end{aligned}
$$

Finally, we calculate the integration over $\Omega(1)$. We further partition $\Omega(1)$ into two regions: $\Omega(1,1)$ consists of all points $x \in \Omega(1)$ whose distance from the boundary of $\Omega$ 
is at most $\frac{r}{2}$, and $\Omega(1,2)=\Omega(1) \backslash \Omega(1,1)$. Then for any $x \in \Omega(1,2)$,

$$
|D(x, r) \cap \Omega| \geq a\left(\frac{r}{2}\right)=\frac{5}{6} \pi r^{2} .
$$

Thus,

$$
\begin{aligned}
& \frac{n}{k !} \int_{\Omega(1,2)}(n|D(x, r) \cap \Omega|)^{k} e^{-n|D(x, r) \cap \Omega|} d x \\
& \leq \frac{n}{k !}\left(n \pi r^{2}\right)^{k} e^{-\frac{5}{6} n \pi r^{2}}|\Omega(1,2)| \\
& =O(1)(n r)\left(n \pi r^{2}\right)^{k} e^{-\frac{5}{6} n \pi r^{2}} \\
& =O(1)\left(\frac{1}{r}\left(n \pi r^{2}\right)^{k} e^{-\frac{1}{2} n \pi r^{2}}\right)\left(\left(n \pi r^{2}\right) e^{-\frac{1}{3} n \pi r^{2}}\right) \\
& =O(1)\left(n \pi r^{2}\right)^{k+1} e^{-\frac{1}{3} n \pi r^{2}}=o(1) .
\end{aligned}
$$

The integration over $\Omega(1,1)$ is calculated as follows. A change of integration variable yields

$$
\begin{aligned}
& \frac{n}{k !} \int_{\Omega(1,1)}(n|D(x, r) \cap \Omega|)^{k} e^{-n|D(x, r) \cap \Omega|} d x \\
& =\frac{4 n(1-2 r)}{k !} \int_{0}^{\frac{r}{2}}(n a(t))^{k} e^{-n a(t)} d t \\
& \sim 4 n \int_{0}^{\frac{r}{2}} \frac{(n a(t))^{k} e^{-n a(t)}}{k !} d t \\
& \sim \frac{\sqrt{\pi}}{2^{k-1} k !} e^{-\frac{\xi}{2}} .
\end{aligned}
$$

In summary, if $k=1$, the integral is asymptotically equal to

$$
e^{-\xi}+\sqrt{\pi} e^{-\frac{\xi}{2}}=e^{-c}
$$

If $k>1$, the integral is asymptotically equal to

$$
\frac{\sqrt{\pi}}{2^{k-1} k !} e^{-\frac{\xi}{2}}=e^{-c}
$$

In either case, Theorem 2 holds.

\subsection{Proof for Theorem 3}

Again by Theorem 1, we only need to show that

$$
\frac{n}{k !} \int_{\Omega}(n|D(x, r) \cap \Omega|)^{k} e^{-n|D(x, r) \cap \Omega|} d x \sim e^{-c} .
$$

To address the boundary effect of the disk region $\Omega$, we partition $\Omega$ into three subregions $\Omega(0), \Omega(1)$ and $\Omega(2)$ as illustrated in Figure 3. Without loss of generality, $\Omega$ is assumed to be centered at the origin o. $\Omega(0)$ is the disk of radius $\frac{1}{\sqrt{\pi}}-r$ centered at $\mathbf{o} ; \Omega(1)$ is the annulus of radii $\frac{1}{\sqrt{\pi}}-r$ and $\sqrt{\frac{1}{\pi}-r^{2}}$ centered at $\mathbf{o}$; and $\Omega(2)$ is the annulus of radii $\sqrt{\frac{1}{\pi}-r^{2}}$ and $\frac{1}{\sqrt{\pi}}$ centered at $\mathbf{o}$. The areas of these three regions are

$$
\begin{aligned}
& |\Omega(0)|=(1-\sqrt{\pi} r)^{2}, \\
& |\Omega(1)|=2 \pi r\left(\frac{1}{\sqrt{\pi}}-r\right), \\
& |\Omega(2)|=\pi r^{2} .
\end{aligned}
$$

For any $x \in \Omega(i)$,

$$
|D(x, r) \cap \Omega| \geq 2^{-i} \pi r^{2} .
$$

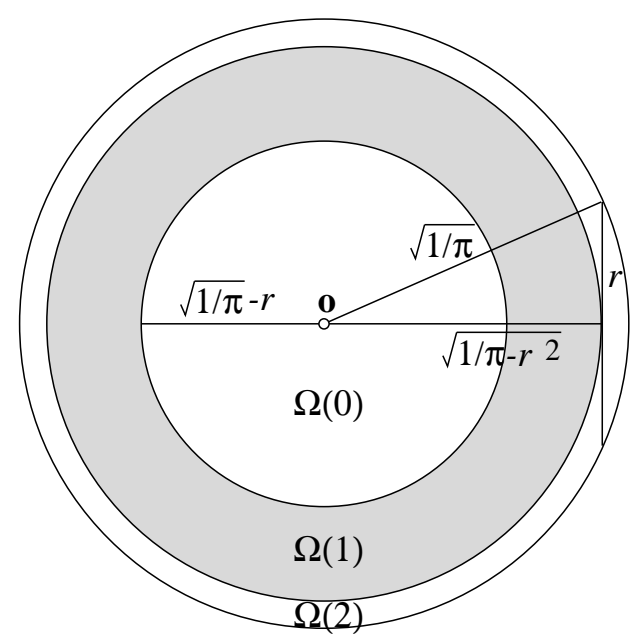

Figure 3: Partition of a disk region $\Omega$.

Using the same argument as in the proof of Theorem 2, we can show that

$$
\begin{aligned}
& \frac{n}{k !} \int_{\Omega(0)}(n|D(x, r) \cap \Omega|)^{k} e^{-n|D(x, r) \cap \Omega|} d x \\
& \sim \begin{cases}e^{-\xi} & \text { if } k=1 \\
o(1) & \text { if } k>1\end{cases}
\end{aligned}
$$

and

$$
\frac{n}{k !} \int_{\Omega(2)}(n|D(x, r) \cap \Omega|)^{k} e^{-n|D(x, r) \cap \Omega|} d x=o(1) .
$$

Next, we calculate the integration over $\Omega(1)$.

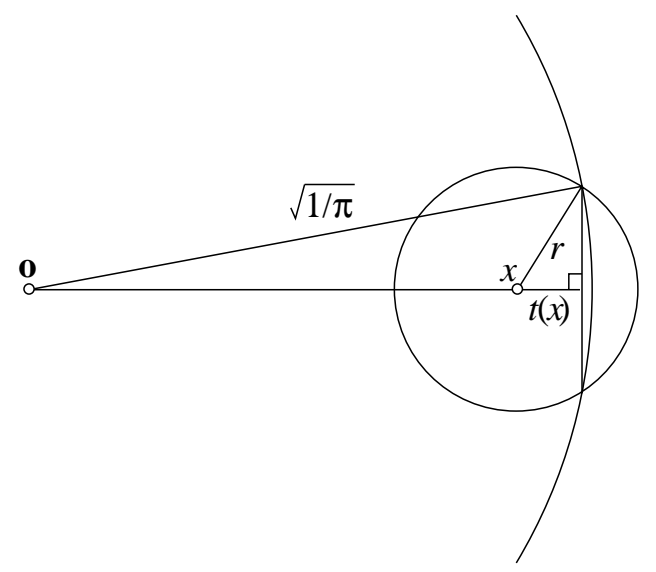

Figure 4: For $x \in \Omega(1), t(x)$ denotes the distance between $x$ and the chord of the circle $\partial D(x, r)$ through the two intersecting points between $\partial D(x, r)$ and $\Omega$. 
For any $x \in \Omega(1)$, let $t(x)$ be the distance between $x$ and the chord of the circle $\partial D(x, r)$ through the two intersecting points between $\partial D(x, r)$ and $\Omega$ (see Figure 4$)$. Then

$$
\|x\|=\sqrt{\frac{1}{\pi}-r^{2}+t(x)^{2}}-t(x) .
$$

In addition,

$$
|D(x, r) \cap \Omega| \geq a(t(x))
$$

and

$$
\begin{aligned}
& |D(x, r) \cap \Omega| \\
& \leq a(t(x))+2 \sqrt{r^{2}-t(x)^{2}}\left(\frac{1}{\sqrt{\pi}}-\sqrt{\frac{1}{\pi}-r^{2}+t(x)^{2}}\right) \\
& =a(t(x))+\frac{2\left(r^{2}-t(x)^{2}\right)^{\frac{3}{2}}}{\frac{1}{\sqrt{\pi}}+\sqrt{\frac{1}{\pi}-r^{2}+t(x)^{2}}} \\
& \leq a(t(x))+2 \sqrt{\pi} r^{3} .
\end{aligned}
$$

Since $a(t(x)) \geq \frac{\pi r^{2}}{2}$, we further have that

$$
\begin{aligned}
& |D(x, r) \cap \Omega| \\
& \leq a(t(x))\left(1+\frac{2 \sqrt{\pi} r^{3}}{a(t(x))}\right) \\
& \leq a(t(x))\left(1+\frac{2 \sqrt{\pi} r^{3}}{\frac{\pi r^{2}}{2}}\right) \\
& =a(t(x))\left(1+\frac{4}{\sqrt{\pi}} r\right) .
\end{aligned}
$$

Thus, for any $x \in \Omega(1)$,

$$
\begin{aligned}
& (n|D(x, r) \cap \Omega|)^{k} e^{-n|D(x, r) \cap \Omega|} \\
& \leq\left(1+\frac{4}{\sqrt{\pi}} r\right)^{k}(n a(t(x)))^{k} e^{-n a(t(x))} .
\end{aligned}
$$

and

$$
\begin{aligned}
& (n|D(x, r) \cap \Omega|)^{k} e^{-n|D(x, r) \cap \Omega|} \\
& \geq e^{-2 \sqrt{\pi} n r^{3}}(n a(t(x)))^{k} e^{-n a(t(x))} .
\end{aligned}
$$

We partition $\Omega(1)$ into two regions: $\Omega(1,1)$ consists of all points $x \in \Omega(1)$ with $t(x) \leq \frac{r}{2}$, and $\Omega(1,2)=\Omega(1) \backslash$ $\Omega(1,1)$. Then for any $x \in \Omega(1,2)$,

$$
|D(x, r) \cap \Omega| \geq a\left(\frac{r}{2}\right)=\frac{5}{6} \pi r^{2} .
$$

Thus, using the same argument as in the proof of Theorem 2 , we can show that

$$
\frac{n}{k !} \int_{\Omega(1,2)}(n|D(x, r) \cap \Omega|)^{k} e^{-n|D(x, r) \cap \Omega|} d x=o(1) .
$$

Finally, we calculate the integration over $\Omega(1,1)$. By the two inequalities just before this theorem,

$$
\begin{aligned}
& \frac{n}{k !} \int_{\Omega(1,1)}(n|D(x, r) \cap \Omega|)^{k} e^{-n|D(x, r) \cap \Omega|} d x \\
& \sim \frac{n}{k !} \int_{\Omega(1,1)}(n a(t(x)))^{k} e^{-n a(t(x))} d x
\end{aligned}
$$

A change of integration variable yields

$$
\begin{aligned}
& \frac{n}{k !} \int_{\Omega(1,1)}(n a(t(x)))^{k} e^{-n a(t(x))} d x \\
& =2 \pi n \int_{0}^{\frac{r}{2}} \frac{f(t)^{k} e^{-f(t)}}{k !}\left(\sqrt{\frac{1}{\pi}-r^{2}+t^{2}}-t\right) \\
& \left(1-\frac{t}{\sqrt{\frac{1}{\pi}-r^{2}+t^{2}}}\right) d t \\
& \left.=2 \pi n \int_{0}^{\frac{r}{2}} \frac{f(t)^{k} e^{-f(t)} \sqrt{\frac{1}{\pi}-r^{2}+t^{2}}}{k !}\right)^{2} d t \\
& \left(1-\frac{t}{\sqrt{\frac{1}{\pi}-r^{2}+t^{2}}}\right)^{\frac{1}{k}} d t \\
& =\frac{\pi}{2^{k} k !} e^{-\frac{\xi}{2}} \cdot
\end{aligned}
$$

Therefore, if $k=1$, the integral is asymptotically equal to

$$
e^{-\xi}+\frac{\pi}{2} e^{-\frac{\xi}{2}}=e^{-c}
$$

If $k>1$, the integral is asymptotically equal to

$$
\frac{\pi}{2^{k} k !} e^{-\frac{\xi}{2}}=e^{-c} \text {. }
$$

In either case, Theorem 3 holds.

\section{CRITICAL NEIGHBOR NUMBER FOR $K$-CONNECTIVITY}

The main result of this section is the following a.a.s. upper bound on $\ell\left(\mathcal{X}_{n} ; \kappa \geq k\right)$.

Theorem 5. For any $k \geq 1$ and $\alpha>1$, the event $\ell\left(\mathcal{X}_{n} ; \kappa \geq k\right) \leq \alpha e \log n$ is a.a.s..

We shall actually prove the following stronger result, which, together with the result by Penrose [13] (for $k=1$ ), Theorem 2 and Theorem 3 (for $k>1$ ), implies Theorem 5 .

TheOrem 6. For any two constants $1<\beta<\alpha$, the event $G \sqrt{\frac{\beta \log n}{\pi n}}\left(\mathcal{X}_{n}\right) \subseteq H_{\alpha e \log n}\left(\mathcal{X}_{n}\right)$ is a.a.s.

Throughout this section, we let $\alpha$ and $\beta$ be fixed constants as in 6 . Pick another constant $\eta \in(\beta, \alpha)$ and let $m$ be the smallest integer which is greater than $1 /(1-\sqrt{\beta / \eta})$. For any integer $n$, let

$$
s_{n}=\sqrt{\frac{\eta \log n}{\pi n}} .
$$


Then

$$
\left(1-\frac{1}{m}\right) s_{n}>\sqrt{\frac{\beta \log n}{\pi n}} .
$$

Let $\mathcal{D}_{n}$ be the set of all open disks of radius $s_{n}$ centered at the square grid of side $\frac{s_{n}}{m}$ with one corner point at the origin which have non-empty intersections with $\Omega$. Let $E_{n}$ denote the event that all disks in $\mathcal{D}_{n}$ contains less than $\alpha e \log n$ nodes of $\mathcal{X}_{n}$.

We first claim that the event $E_{n}$ implies the event that $G_{\sqrt{\frac{\beta \log n}{\pi n}}}\left(\mathcal{X}_{n}\right) \subseteq H_{\alpha e \log n}\left(\mathcal{X}_{n}\right)$. Assume that then event $E_{n}$ occurs. For any node $X \in \mathcal{X}_{n}$, there exist a disk $D^{*}$ in $\mathcal{D}_{n}$ such that the distance between $X$ and the center of $D$ is less than $s_{n} / m$. Thus,

$$
D\left(X, \sqrt{\frac{\beta \log n}{\pi n}}\right) \subset D\left(X,\left(1-\frac{1}{m}\right) s_{n}\right) \subset D^{*} .
$$

Since $D^{*}$ contains less than $\alpha e \log n$ nodes of $\mathcal{X}_{n}$, so does the disk $D\left(X, \sqrt{\frac{\beta \log n}{\pi n}}\right)$. This implies that any neighbor of $X$ in $G \sqrt{\frac{\beta \log n}{\pi n}}\left(\mathcal{X}_{n}\right)$ is one of its $\alpha e \log n$ nearest neighbors. Now consider any edge $X Y$ in $G \sqrt{\frac{\beta \log n}{\pi n}}\left(\mathcal{X}_{n}\right)$. Then both $X$ and $Y$ are one of each other's $\alpha e \log n$ nearest neighbors. Consequently, $X Y$ is also an edge of $H_{\alpha e \log n}\left(\mathcal{X}_{n}\right)$. So our claim is true. Therefore, Theorem 6 would follows if we can prove that $E_{n}$ is an a.a.s. event. The remaining of this section is devoted to this proof.

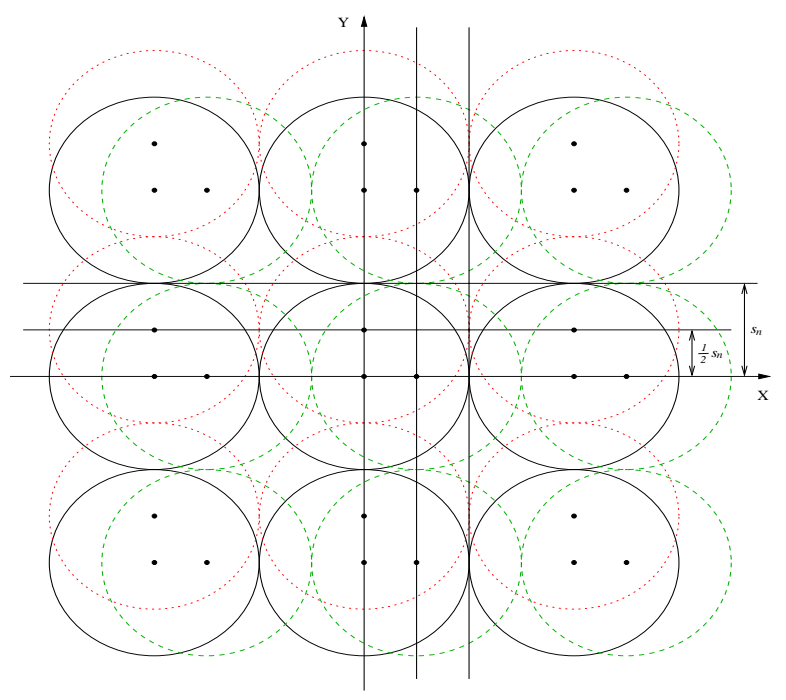

Figure 5: For $m=2$, disks with black solid line form $\mathcal{D}_{n}^{00}$, disks with green dash line form $\mathcal{D}_{n}^{10}$, and disks with red dot line form $\mathcal{D}_{n}^{01}$.

We partition $\mathcal{D}_{n}$ into $(2 m)^{2}$ subsets $\mathcal{D}_{n}^{i j}$ with $0 \leq i, j<$ $2 m$ where $\mathcal{D}_{n}^{i j}$ consists of all disks in $\mathcal{D}_{n}$ centered at the square grid of side $2 s_{n}$ with one corner point at $\left(i \frac{s_{n}}{m}, j \frac{s_{n}}{m}\right)$. See Figure 5. Correspondingly, for any $0 \leq i, j<2 m$ let
$E_{n}^{i j}$ denote the event that all disks in $\mathcal{D}_{n}^{i j}$ contains less than $\alpha e \log n$ nodes of $\mathcal{X}_{n}$. Then

$$
\mathcal{D}_{n}=\bigcup_{i=0}^{2 m-1} \bigcup_{i=0}^{2 m-1} \mathcal{D}_{n}^{i j}, E_{n}=\bigcap_{i=0}^{2 m-1} \bigcap_{i=0}^{2 m-1} E_{n}^{i j} .
$$

Since the intersection of a constant number of a.a.s. events is also an a.a.s. event, it is sufficient to show that each $E_{n}^{i j}$ is an a.a.s. event. We prove this by using the same Poissonization technique as in [20]. Fix two integers $0 \leq i, j<2 m$. We denote by $\widetilde{E}_{n}^{i j}$ the event that all disks in $\mathcal{D}_{n}^{i j}$ contains less than $\eta e \log n$ nodes of $\mathcal{P}_{n}$. Since $\eta<\alpha$, using the similar proof of Lemma 3.2.3 in [20] we can show that if $\widetilde{E}_{n}^{i j}$ is an a.a.s. event, so must be $E_{n}^{i j}$. Thus, we only to prove that $\widetilde{E}_{i j}$ is an a.a.s. event. To prove this, we number the disks in $\mathcal{D}_{n}^{i j}$ by

$$
\mathcal{D}_{n}^{i j}=\left\{D_{t}: t \in I\right\},
$$

where $I$ is the index set. For any $t \in I$, let $N_{t}$ be the number of points of $\mathcal{P}_{n}$ which fall in $D_{t}$. Then $\widetilde{E}_{n}^{i j}$ can be expressed as $\max _{t \in I} N_{t}<\eta e \log n$. In the next, we show that

$$
\operatorname{Pr}\left\{\max _{t \in I} N_{t}<\eta e \log n\right\} \sim 1 \text {. }
$$

Let $\lambda=n \cdot \pi s_{n}^{2}=\eta \log n$ and $M$ be a Poisson random variable with rate $\lambda$. The following upper bound on the tail distribution of $M$ follows from Lemma 3.2.5 in [20] and Stirling's formula:

$$
\begin{aligned}
& \operatorname{Pr}\{M \geq e \lambda\} \\
& =\frac{e}{e-1} \frac{\lambda^{e \lambda}}{(e \lambda) !} e^{-\lambda}(1+o(1)) \\
& \leq \frac{e}{e-1} \frac{\lambda^{e \lambda}}{\sqrt{2 \pi e \lambda}\left(\frac{e \lambda}{e}\right)^{e \lambda}} e^{-\lambda}(1+o(1)) \\
& =\frac{e}{e-1} \frac{e^{-\lambda}}{\sqrt{2 \pi e \lambda}}(1+o(1)) \\
& =\Theta\left(\frac{1}{n^{\eta} \sqrt{\log n}}\right) .
\end{aligned}
$$

This bound implies that $\operatorname{Pr}\{M \geq e \lambda\}=o(1)$. Furthermore, since

$$
\operatorname{card}(I)=\Theta\left(\frac{1}{\pi s_{n}^{2}}\right)=\Theta\left(\frac{n}{\log n}\right)
$$

we have

$$
\begin{aligned}
& \operatorname{card}(I) \operatorname{Pr}\{M \geq e \lambda\} \\
& \leq \Theta\left(\frac{n}{\log n} \cdot \frac{1}{n^{\eta} \sqrt{\log n}}\right) \\
& =\Theta\left(\frac{1}{n^{\eta-1}(\log n)^{3 / 2}}\right) \\
& =o(1) .
\end{aligned}
$$

For any $t \in I$, let $\lambda_{t}=n\left|D_{t} \cap \Omega\right|$. Then each $N_{t}$ is a Poisson random variable with rate $\lambda_{t}$. Note that for any integer $q$, the function $f_{q}(\mu)=\mu^{q} e^{-\mu}$ is strictly increasing as long as $0<\mu<q$, since

$$
f_{q}^{\prime}(\mu)=q \mu^{q-1} e^{-\mu}-\mu^{q} e^{-\mu}=\mu^{q-1} e^{-\mu}(q-\mu)
$$


As $\lambda_{t} \leq \lambda=\eta \log n$, we have

$$
\begin{aligned}
& \operatorname{Pr}\left\{N_{t} \geq \eta e \log n\right\}=\operatorname{Pr}\left\{N_{t} \geq e \lambda\right\} \\
& =\sum_{q=\lceil e \lambda\rceil}^{\infty} \frac{\lambda_{t}^{q}}{q !} e^{-\lambda_{t}} \leq \sum_{q=\lceil e \lambda\rceil}^{\infty} \frac{\lambda^{q}}{q !} e^{-\lambda} \\
& =\operatorname{Pr}\{M \geq e \lambda\} .
\end{aligned}
$$

This inequality together with the independence of $\mathcal{P}_{n}$ implies that

$$
\begin{aligned}
& \operatorname{Pr}\left\{\max _{t \in I} N_{t}<\eta e \log n\right\}=\operatorname{Pr}\left\{\max _{t \in I} N_{t}<e \lambda\right\} \\
& =\prod_{t \in I} \operatorname{Pr}\left\{N_{t}<e \lambda\right\} \\
& =\prod_{t \in I}\left(1-\operatorname{Pr}\left\{N_{t} \geq e \lambda\right\}\right) \text {. } \\
& \geq \prod_{i \in I}(1-\operatorname{Pr}\{M \geq e \lambda\}) \\
& =(1-\operatorname{Pr}\{M \geq e \lambda\})^{\operatorname{card}(I)} \\
& =\left((1-\operatorname{Pr}\{M \geq e \lambda\})^{\frac{1}{\operatorname{Pr}\{M \geq e \lambda\}}}\right)^{\operatorname{card}(I) \operatorname{Pr}\{M \geq e \lambda\}} \\
& \sim e^{-\operatorname{card}(I) \operatorname{Pr}\{M \geq e \lambda\}} \\
& \sim 1 \text {. }
\end{aligned}
$$

Therefore, each $\widetilde{E}_{n}^{i j}$ is an a.a.s. event. This completes the proof of Theorem 6 .

\section{CONCLUSION}

In this paper, we model the wireless ad hoc network by a uniform $n$-point process $\mathcal{X}_{n}$ over a unit-area disk or square $\Omega$. We derived the precise asymptotic distribution of the critical transmission radius for $k$-connectivity $\rho\left(\mathcal{X}_{n} ; \kappa \geq k\right)$. We also obtained an improved asymptotic almost sure upper bound on the critical neighbor number for $k$-connectivity $\ell\left(\mathcal{X}_{n} ; \kappa \geq k\right)$.

\section{REFERENCES}

[1] Bettstetter, C. On the minimum node degree and connectivity of a wireless multihop network. In Proceedings of the 3rd ACM International Symposium on Mobile Ad Hoc Networking and Computing (MobiHoc'02) (June 9-11 2002), pp. 80-91.

[2] Cheng, Y.-C., and Robertazzi, T. G. Critical connectivity phenomena in multihop radio models. IEEE Transactions on Communications 37, 7 (July 1989), 770-777.

[3] Dette, H., And Henze, N. The limit distribution of the largest nearest-neighbour link in the unit $d$-cube. Journal of Applied Probability 26 (1989), 67-80.

[4] Dette, H., And Henze, N. Some peculiar boundary phenomena for extremes of $r$ th nearest neighbor links. Statistics $\&$ P Probability Letters 10 (1990), 381-390.

[5] Dousse, O., Thiran, P., and Hasler, M. Connectivity in ad-hoc and hybrid networks. In Proceedings of the 21st Annual Joint Conference of the IEEE Computer and Communications Societies (IEEE
INFOCOM 2002) (June 23-27 2002), vol. 2, pp. 1079-1088.

[6] Gupta, P., And Kumar, P. R. Critical power for asymptotic connectivity in wireless networks. In Stochastic Analysis, Control, Optimization and Applications: A Volume in Honor of W. H. Fleming (March 1998), W. M. McEneaney, G. Yin, and Q. Zhang, Eds., Birkhauser, pp. 547-566.

[7] HAJEK, B. Adaptive transmission strategies and routing in mobile radio networks. In Proceedings of the Conference Information Sciences and Systems (March 1983), pp. 373-378.

[8] Hou, T.-C., And Li, V. O. Transmission range control in multihop packet radio networks. IEEE Transactions on Communications COM-34, 1 (January 1986), 38-44.

[9] Kleinrock, L., And Silvester, J. A. Optimum transmission radii for packet radio networks or why six is a magic number. In IEEE Nat. Telecommun. Conf. (December 1978), pp. 4.3.1-4.3.5.

[10] Krishnamachari, B., Wicker, S. B., and BÉJar, $\mathrm{R}$. Phase transition phenomena in wireless ad hoc networks. In IEEE Global Telecommunications Conference (GLOBECOM '01) (Nov. 25-29 2001), vol. 5, pp. 2921-2925.

[11] Mathar, R., And Mattfeldt, J. Analyzing routing strategy NFP in multihop packet radio networks on a line. IEEE Transactions on Communications 43, 234 (February/March/April 1995), 977-988.

[12] Ni, J., AND Chandler, S. Connectivity properties of a random radio network. In Proceedings of the IEE proceedings - Communications (August 1994), vol. 141, pp. 289-296.

[13] Penrose, M. D. The longest edge of the random minimal spanning tree. The annals of applied probability 7, 2 (1997), 340-361.

[14] Penrose, M. D. On $k$-connectivity for a geometric random graph. Random Structures and Algorithms 15, 2 (September 1999), 145-164.

[15] Piret, P. On the connectivity of radio networks. IEEE Transactions on Information Theory 37, 5 (September 1991), 1490-1492.

[16] Santi, P., And Blough, D. M. An evaluation of connectivity in mobile wireless ad hoc networks. In Proceedings of the International Conference on Dependable Systems and Networks (DSN 2002) (June 23-26 2002), IEEE, pp. 89-98.

[17] Silvester, J. A. On the spatial capacity of packet radio networks. Tech. Rep. Eng. Rep. UCLA-ENG-8021, Dept. Comput. Sci., School Eng. Appl. Sci., Univ. California, Los Angeles, May 1980.

[18] TAKagi, H., And Kleinrock, L. Optimal transmission ranges for randomly distributed packet radio terminals. IEEE Transactions on Communications COM-32, 3 (March 1984), 246-257.

[19] Wang, J. L., And Silvester, J. A. Maximum number of independent paths and radio connectivity. IEEE Transactions on Communications 41, 10 (October 1993), 1482-1493.

[20] Xue, F., And Kumar, P. R. The number of neighbors needed for connectivity of wireless networks. Wireless Networks 10, 2 (March 2004), 169-181. 